\title{
"Escuela de Cuidadores" como programa psicoeducativo para cuidadores informales de adultos mayores con demencia
}

\section{School of caregivers - a psychoeducational program for informal caregivers of demented older people}

\section{Ana Margarita Espín Andrade}

Máster en Psicología de la Salud. Asistente. Escuela Latinoamericana de Medicina (ELAM). La Habana, Cuba.

\section{RESUMEN}

I ntroducción La demencia es una enfermedad que afecta tanto al paciente como a la familia y en particular al cuidador, que es la persona que dentro de ella, asume la mayor responsabilidad en el cuidado. Existen diferentes modalidades de intervención que mejoran la calidad del cuidado al paciente y reducen la carga del cuidador.

Objetivos Diseñar y evaluar el proceso y la efectividad de un programa de intervención psicoeducativo en un grupo de cuidadores informales de adultos mayores con demencia.

Métodos Se realizó un estudio pre-experimental con 16 cuidadores que recibieron atención en el Centro I beroamericano para la Tercera Edad, de 2004 a 2005. Se diseñó el programa a partir de técnicas participativas de recogida de información con expertos y con cuidadores e información actualizada del tema; se conformó con 10 sesiones y una frecuencia semanal. Se aplicó la Escala Psicosocial del Cuidador para constatar las diferencias entre antes y después de la intervención con respecto a variables socioeconómicas y psicológicas. Los datos se procesaron y analizaron 
mediante la prueba McNemar para dos variables cualitativas relacionadas, fijándose la significación estadística en $\alpha \leq 0,05$.

Resultados Disminuyeron en general las afectaciones socioeconómicas y psicológicas del cuidador después de la intervención, como son: el poco tiempo libre, las dificultades laborales, los sentimientos de angustia, ira y miedo pero en el caso de las variables socioeconómicas la mejoría no fue significativa.

Conclusiones Se demuestra que el programa psicoeducativo "Escuela de Cuidadores"es efectivo para modificar la afectación psicológica de los cuidadores, pero no para mejorar las afectaciones de tipo socioeconómica que produce el cuidado de un anciano con demencia, lo que indica que este tipo de intervención no es suficiente para modificar los aspectos más tangibles de la vida del cuidador debiendo complementarse con otras intervenciones psicosociales.

Palabras clave: Demencia, cuidador informal, programa intervención, intervención psicoeducativa.

\section{ABSTRACT}

Introduction Dementia is a disease that affects the patient, the family, and particularly the caregiver who is the main responsible for the care of that person. There are different intervention modalities that improve the quality of care to the patient and reduce the caregiver's burden.

Objectives to design and evaluate the process and effectiveness of a psychoeducational intervention program in a group of informal caregivers of demented older adults.

Methods A pre-experimental study was made on 16 caregivers, who were linked to the Iberoamerican Center for the Elderly, from 2004 to 2005. A program was designed on the basis of participatory techniques of data gathering with experts and with caregivers, and updated information on this topic; it was organized in 10 sessions that were held once a week. The Psychosocial Scale of Caregiver was used to compare the differences before and after the intervention in terms of socioeconomic and psychological variables. McNemar's test was the choice for data processing and analysis of two related qualitative variables whereas significance statistics was set as $\alpha \leq 0.05$.

Results Psychological and socioeconomic effects on the caregivers decreased in general after the intervention, namely, feelings of anger, anguish and fear, little leisure time, and unmet needs; however, the improvement was not significant in some socioeconomic variables.

Conclusions It was shown that the psychoeducational program "School of Caregivers" was effective in changing the psychological effects on the caregivers but not in reducing all the socioeconomic effects caused by the care of a demented elder; this indicates that this type of intervention is not enough to modify the most tangible aspects of the caregiver's life, which requires other supplementary psychosocial interventions.

Key words: Dementia, informal caregiver, intervention program, psychoeducational intervention.

\section{NTRODUCCI ÓN}


La demencia es una enfermedad del sistema nervioso central que afecta los procesos mentales superiores del individuo como la memoria, el lenguaje, la atención, el pensamiento, y provoca alteraciones en la conducta y la personalidad de las personas que la padecen. Se clasifica en varios tipos siendo más común la enfermedad de Alzheimer seguida de las demencias vasculares. Su aparición es más frecuente después de los 60 años es decir se incrementa con la edad por lo que su prevalencia ha aumentado con el envejecimiento poblacional en los últimos años y seguirá creciendo.

Las enfermedades demenciales, en general, afectan entre 18 y 22 millones de personas a nivel mundial. Esta cifra llegará a 40 millones de personas en el año 2025, fecha para la cual la población mayor de 65 años se duplicará de 390 millones a $800 .^{1}$

Se calcula que aproximadamente 100000 personas en Cuba la padecen. Un estudio realizado en un municipio de Ciudad Habana encuentra una prevalencia de 5,42 \%, y es la enfermedad de Alzheimer la causa más frecuente con 81 pacientes para el $70 \% .^{2}$

El costo económico de la enfermedad de Alzheimer es muy elevado, siendo la tercera enfermedad más costosa detrás de los problemas cardíacos y del cáncer. Estos costos son especialmente elevados cuando existen condiciones comórbidas al Alzheimer (el $63 \%$ de los enfermos de Alzheimer tienen además otras enfermedades). ${ }^{3}$

La demencia provoca una pérdida progresiva de la autonomía y el validismo del enfermo el que se torna dependiente de las demás personas para realizar sus actividades de la vida diaria. Esta dependencia unida a los problemas conductuales como delirios, alucinaciones, agresividad, desinhibición y otros síntomas neuropsiquiátricos, conllevan a la aparición de alteraciones en la dinámica familiar y en particular en la calidad de vida de los cuidadores que son aquellas personas que dentro de ella asumen la responsabilidad en su atención.

La familia es la principal proveedora de cuidados al adulto mayor dependiente. En estudios realizados se ha encontrado que el $71,9 \%$ de los cuidadores de personas mayores con Alzheimer son miembros de la familia. ${ }^{4}$

Se han estudiado las características generales de los cuidadores informales de personas que sufren algún grado de dependencia por diferentes tipos de enfermedad y se ha hallado coincidencia en que son mujeres entre 40 y 59 años, hijas del enfermo, que conviven generalmente con este, casadas y con hijos. También se ha descrito que sufren afectaciones físicas, psicológicas y sociales, lo cual conlleva un sentimiento de sobrecarga. ${ }^{5-8}$

Si bien esto es así, se asegura que el cuidado de personas con demencia es mucho más estresante que atender a personas con dependencia física o con muy pocos problemas emocionales o conductuales. ${ }^{9,10}$ Esto al parecer se debe a que el cuidador del paciente con demencia debe introducir mayores cambios en su estilo de vida, tiene una mayor limitación social, sufre una alteración de la relación interpersonal cuidador-enfermo y disminuye la gratificación por parte del receptor de los cuidados.

Numerosas variables inciden en la carga entre las que se encuentran las relacionadas con el paciente: años de duración de la enfermedad y problemas conductuales del paciente (agresividad, incontinencia, vagabundeo, acusaciones, 
apatía), las propias del cuidador: estilos de afrontamiento (coping), recursos financieros, apoyo social (emocional), el número de roles y por último las derivadas de la relación cuidador-paciente, asegurando que a mayor implicación emocional mayor carga.

El cuidador presenta una serie de alteraciones físicas y de su salud como son las quejas somáticas, el dolor crónico del aparato locomotor, la cefalea tensional, la astenia y la fatiga crónica, la alteración del ciclo sueño-vigilia, el deterioro de la función inmune y una mayor predisposición a úlcera péptica y a enfermedades cardiovasculares, entre otras.

Por otra parte, el cuidador puede presentar problemas psíquicos como depresión, ansiedad, insomnio, y una alta tasa de automedicación. Los problemas de índole socioeconómicos son otro grupo de alteraciones que puede presentar el cuidador de un enfermo con demencia. Estos son conflictos familiares, problemas laborales, dificultades económicas y disminución de actividades sociales y de ocio.

Según la Asociación de Familiares de Alzheimer (AFA), el sistema familiar en su conjunto padece la enfermedad de Alzheimer, en el caso de que esta se produzca y aconseja que dicha familia o el cuidador del afectado, se informe sobre tal enfermedad así como de su evolución, fases clínicas, sintomatología más característica, precauciones que deben de tomar, necesidades asistenciales y algún consejo práctico para el cuidado de estas personas enfermas. ${ }^{11}$

Por todo lo planteado se hace necesario llevar a cabo acciones encaminadas a elevar la calidad de vida de estos cuidadores lo cual a su vez redundará en un cuidado mejor y en el mayor bienestar de la familia.

Las intervenciones tienen como objetivo disminuir la carga que experimentan las personas que cuidan a ancianos dependientes, el estrés y malestar asociados al cuidado y por otra parte mejorar la calidad de los cuidados que reciben estos ancianos. Para conseguir estos objetivos se han empleado procedimientos de intervención psicosocial tales como grupos de educación e información, grupos de apoyo emocional y autoayuda, grupos de entrenamiento en técnicas cognitivoconductuales y tratamientos individuales como asesoramiento familiar, consulta familiar o terapia psicológica individual. ${ }^{12}$

La intervención en educación y el consejo o instrucción a los cuidadores para solucionar problemas específicos, mejoran y estabilizan más la función familiar que los programas habituales de seguimiento y tratamiento. ${ }^{13}$

Entre las modalidades de intervención se encuentran los programas psicoeducativos que tienen el propósito de ofrecer en primer lugar información acerca de la enfermedad y su manejo así como brindar estrategias de afrontamiento y apoyo emocional a los cuidadores.

Las intervenciones a través de las cuales se obtienen mejores resultados son las psicoeducativas y las psicoterapéuticas, exitosas ambas a la hora de aliviar la carga y la depresión de los cuidadores, efectos que se mantienen durante un período considerable. $^{7}$

En un estudio realizado en 158 cuidadores se evalúa la eficacia de un programa psicoeducativo y se encuentra una disminución de $14 \%$ en las reacciones emocionales negativas a los problemas de conducta del enfermo. ${ }^{14}$ 
Los programas que sólo brindan información a los cuidadores puede generarles un alto grado de ansiedad, lo cual se ha relacionado con una anticipación de la pérdida y con el conocimiento del alcance de la enfermedad, por lo que es necesario ofrecerles ayuda para procesar la información de tal forma que sus emociones sean más adecuadas. ${ }^{15}$

A pesar de que se realizan numerosas intervenciones grupales e individuales en los cuidadores son aún muy pocos los estudios que las evalúan lo cual es muy importante porque permite brindar una ayuda y preparación más eficaz a estas personas.

Son múltiples las propuestas para intervenir con estos cuidadores familiares pero muy pocas las que se han realizado en el contexto regional y menos aún aquellas que han sido evaluadas con rigor. ${ }^{16}$

El presente trabajo tiene el objetivo de describir y evaluar el proceso y la efectividad, de un programa de intervención psicoeducativo en un grupo de cuidadores informales de adultos mayores con demencia.

\section{MÉTODOS}

\section{Universo}

Conformado por 60 cuidadores informales de pacientes con demencia diagnosticados en el Centro I beroamericano para la Tercera Edad, de diciembre de 2004 a diciembre del 2005.

\section{Muestra}

Constituida por 16 cuidadores informales seleccionados aleatoriamente para participar en el programa de intervención. El tamaño de la muestra se determinó por el criterio de que los grupos que participan no deben ser muy numerosos porque es necesario garantizar la interacción de los miembros dentro del grupo.

\section{Aspectos éticos}

Se pidió y obtuvo el consentimiento de los cuidadores seleccionados y la totalidad mostró disposición y posibilidad de participar en el programa.

\section{Tipo de diseño}

Se realizó un estudio pre-experimental puesto que se realizó una evaluación al grupo de cuidadores antes y después de su participación en el programa de intervención psicoeducativo, sin utilizar grupo control.

\section{Técnicas y procedimientos}

Para el diseño del programa "Escuela de Cuidadores"se utilizaron las técnicas de grupo focal y grupo nominal con el objetivo de identificar las necesidades reales y sentidas de los cuidadores que conformaron la muestra. Para la búsqueda de los antecedentes acerca de este tipo de intervención y las opiniones de expertos en la 
identificación de los temas a tratar, se utilizó como fuente de información la literatura científica teniendo en cuenta los objetivos del programa.

\section{Temas que conforman el programa de intervención psicoeducativo}

Se diseñó un programa psicoeducativo conformado con los siguientes temas:

- Características de la demencia.

- Manejo de los hábitos de vida: alimentación, higiene personal y sueño.

- Manejo de problemas de la conducta.

- Comunicación.

- Actividad física y autonomía.

- Estimulación cognitiva.

- Cuidados del cuidador, prevención de complicaciones.

- Manejo de la carga del cuidador.

El programa se organizó con diez sesiones y una frecuencia semanal. Las sesiones se realizaron por parte de un equipo de profesionales conocedores de las diferentes temáticas: geriatras, psicólogos, enfermeras geriátricas y trabajadores sociales.

\section{Objetivos y evaluación del programa de intervención psicoeducativo}

Los objetivos del programa son los siguientes:

1. Trasmitir conocimientos acerca de las demencias y el manejo adecuado de sus manifestaciones conductuales y síntomas psiquiátricos.

2. Desarrollar habilidades con respecto al manejo de los hábitos de vida del enfermo.

3. Brindar soporte emocional a los cuidadores.

Se establecieron los criterios y los estándares para la evaluación del programa de intervención a los cuales se llegaron a través de la opinión de expertos. Estos fueron los siguientes:

1. Porcentaje de permanencia de los cuidadores en la intervención.

2. Porcentaje de asistencia.

3. Porcentaje de participación de los cuidadores en las sesiones.

4. Evaluación cualitativa de las sesiones en muy bien, bien, regular y mala. ( MB, B, $R, M$, respectivamente). 
La evaluación de efectividad se realizó a través de la posible modificación de las variables socioeconómicas y psicológicas de los cuidadores a partir de la Escala Psicosocial del cuidador. Esta es una escala likert que mide dos tipos de variables; las socioeconómicas entre las que se encuentran las siguientes: recursos de apoyo, tiempo libre, recursos económicos, satisfacción de necesidades, conflictos familiares, relaciones sociales y dificultades laborales; y las variables psicológicas donde se encuentran: angustia, irritabilidad, vergüenza, culpabilidad, miedo, soledad, resentimiento y desesperanza.

Se utilizó el método test-retest aplicando la escala psicosocial antes y después de la intervención y se analizó la diferencia entre los resultados de cada una de las variables en ambos momentos.

\section{Procedimiento estadístico}

Se confeccionó la base de datos y se procesaron y analizaron los datos a través de la prueba McNemar para dos variables cualitativas relacionadas. La significación estadística se fijó en $\alpha \leq 0,05$.

\section{RESULTADOS}

El grupo de 16 cuidadores informales seleccionados tenía las siguientes características que fueron recogidas a través del cuestionario de caracterización del cuidador. ${ }^{17}$

- Edad: de 40 a 59: 11; +60 años: 5.

- Sexo: F: 9; M: 7.

- Estado civil: Casados: 11; Solteros: 5.

- Escolaridad: Primaria: 1; Media: 3; Universitaria: 12.

- Ocupación: Trabajadores: 10; Jubilados: 4; Amas de casa: 2.

- Parentesco: Hijos: 14; Cónyuges: 1; Nietos: 1.

- Número de personas a cuidar: una: 8; dos: 7; más de dos: 1.

- Motivación para el cuidado: Lazos afectivos: 13; Interés material: 2; Deber moral: 1.

- Información previa sobre la enfermedad: Nula: 11; Insuficiente: 5.

- Problemas de salud presentados: Óseos: 5; Cardíacos: 5; Hipertensión arterial: 3; Nerviosos: 2.

- Estrategia de afrontamiento: Búsqueda de apoyo de familiares: 5; Búsqueda de información: 5; Búsqueda de ayuda de otras personas: 6 . 
- Tiempo de cuidador: menos de 1 año: 8; de 1 a 4 años: 3; más de 4 años: 5.

En la evaluación de proceso se encontró que el $80 \%$ de los cuidadores llegó al final de la intervención, hubo un promedio de asistencia de $94 \%$, el promedio de participación por sesión fue de $75 \%$ y las sesiones fueron evaluadas de MB y B por el $100 \%$ de los cuidadores.

Con respecto a la evaluación de la efectividad, se observó que casi la totalidad de las variables socioeconómicas disminuyeron después de la intervención, aunque su variación no fue significativa, valores de $p$ mayores de 0,05 (tabla 1 ).

Tabla 1. Efectividad del programa atendiendo a variables socioeconómicas

\begin{tabular}{|c|c|c|c|c|c|}
\hline \multirow{2}{*}{$\begin{array}{c}\text { Variables } \\
\text { socioeconómicas }\end{array}$} & \multicolumn{2}{|c|}{ Antes } & \multicolumn{2}{|c|}{ Después } & \multirow[t]{2}{*}{ Valor de p* } \\
\hline & No. & $\%$ & No. & $\%$ & \\
\hline $\begin{array}{l}\text { Apoyo } \\
\text { insuficiente }\end{array}$ & 8 & 50 & 7 & 44 & 0,70 \\
\hline Poco tiempo libre & 15 & 94 & 13 & 81 & 0,15 \\
\hline $\begin{array}{l}\text { Problemas } \\
\text { económicos }\end{array}$ & 9 & 56 & 8 & 50 & 0,56 \\
\hline $\begin{array}{l}\text { Insatisfacción de } \\
\text { necesidades }\end{array}$ & 9 & 56 & 9 & 56 & 1,00 \\
\hline $\begin{array}{l}\text { Conflictos } \\
\text { familiares }\end{array}$ & 12 & 75 & 11 & 69 & 0,56 \\
\hline $\begin{array}{l}\text { Pocas relaciones } \\
\text { sociales }\end{array}$ & 10 & 63 & 9 & 56 & 0,70 \\
\hline $\begin{array}{l}\text { Dificultades } \\
\text { laborales }\end{array}$ & 9 & 56 & 7 & 44 & 031 \\
\hline
\end{tabular}

* Prueba de McNemar, $\alpha \leq 0,05$.

Según los resultados de la evaluación de efectividad atendiendo a las variables psicológicas se observó que todas, excepto el resentimiento, se modificaron favorablemente después de la intervención pero sólo fueron significativos la angustia, la ira y el miedo con valores de $p \leq 0,05$ (tabla 2). 
Tabla 2. Efectividad del programa atendiendo a variables psicológicas

\begin{tabular}{|l|c|c|c|c|c|}
\hline \multirow{2}{*}{\begin{tabular}{|l|l|l|l|} 
Variables \\
psicológicas
\end{tabular}} & \multicolumn{2}{|c|}{ Antes } & \multicolumn{2}{c|}{ Después } & Valor de p* \\
\cline { 2 - 3 } & No. & $\%$ & No. & $\%$ & \\
\hline Angustia & 12 & 75 & 9 & 56 & 0,05 \\
\hline Ira & 14 & 88 & 9 & 56 & 0,04 \\
\hline Miedo & 8 & 50 & 7 & 44 & 0,65 \\
\hline Soledad & 5 & 31 & 3 & 19 & 0,15 \\
\hline Culpa & 3 & 19 & 3 & 19 & 1,00 \\
\hline Resentimiento & & & & & 0,05 \\
\hline Desesperanza & 10 & 63 & 7 & 44 & 0,25 \\
\hline
\end{tabular}

* Prueba de McNemar, $\alpha \leq 0,05$.

\section{DISCUSIÓN}

Si bien es abundante la literatura existente acerca de las características de los cuidadores y de la afectación de diversa índole que estos sufren por el cuidado de una persona mayor dependiente, en particular con demencia, son insuficientes los estudios publicados acerca de programas de intervención diseñados a partir de las necesidades de los cuidadores dirigidos a mejorar los aspectos psicológicos y socioeconómicos de la vida del cuidador por lo que la discusión del presente trabajo se basa fundamentalmente en los resultados obtenidos.

A pesar de haber encontrado afectación de prácticamente todas las variables psicológicas y socioeconómicas medidas, pudiera existir un sesgo en la información debido a que los instrumentos que se utilizaron eran de autoreporte donde los cuidadores reconocen por lo general menos carga de la que realmente tienen. Esto coincide con lo planteado por algunos autores acerca de que aunque resulta paradójico no siempre los cuidadores están dispuestos a reconocer el sufrimiento emocional que padecen. Es relativamente frecuente que el cuidador niegue soportar un peso superior a sus fuerzas y se oponga a que alguien dude de su capacidad física y psíquica para ocuparse del cuidado de su familiar. ${ }^{18}$

Entre los principales problemas, encontrados en la totalidad de los cuidadores y que están muy relacionados, estaba el poco tiempo libre con que contaban y la insatisfacción de sus necesidades personales. Al parecer los cuidadores aprendieron a planificar mejor su tiempo libre y a darle a este el valor que tiene y su repercusión en el ofrecimiento de un cuidado de mayor calidad. En relación con esta variable se ha señalado que debido al vínculo de dependencia que crea el familiar afecto de demencia con su cuidador, este va perdiendo poco a poco su independencia, se olvida de sí mismo y no se toma el tiempo libre que necesita 
para descansar, abandona sus hobbies y aficiones, deja de comunicarse con los amigos y acaba finalmente paralizando durante algunos años su proyecto de vida.

Un aspecto que sufrió ligeros cambios con la intervención y que afectaba a la mitad de los cuidadores era el insuficiente apoyo recibido por parte de otros familiares y de otras personas para el cuidado del enfermo. Esto pudiera deberse a la tendencia de los cuidadores planteada anteriormente a no pedir ayuda por considerarlo una acción de incapacidad y de rechazo al enfermo.

Otro problema encontrado en un elevado número de cuidadores que se modificó discretamente fue la presencia de conflictos en la familia ocasionados a partir de la enfermedad del anciano. Esto pudiera deberse a la no intervención psicoeducativa en la familia como un todo sino tan sólo en el cuidador lo que sugiere la necesidad de la incorporación del resto de los familiares a este programa.

En un estudio realizado se aprecia que hay una menor sobrecarga en el cuidador cuando este recibe el apoyo social de sus familiares allegados, vecinos y otros, mientras que los que no lo reciben, son más propensos a padecer depresión y a sentir angustia. ${ }^{19}$ Sería conveniente incorporar al programa de intervención a otros familiares cercanos al enfermo con el objetivo de involucrarlos y prepararlos directamente para su cuidado.

La demencia es un asunto de la familia en su conjunto y todos tienen su papel y responsabilidad en el cuidado de su familiar. Sería óptimo que existiese entre ellos una comunicación abierta, con el propósito de repartir a cada miembro de la familia sus respectivas tareas de cuidado. Por otra parte, deben ponerse de acuerdo en las decisiones y manejo de las actividades y problemas del enfermo, lo que favorece la armonía y coherencia entre ellos que permite un cuidado más satisfactorio y una atmósfera más positiva para el paciente y para el cuidador principal. Estos son elementos que al no cumplirse repercuten en la aparición de conflictos familiares que se observaron en un elevado número de cuidadores, aspecto que mejoró discretamente con la intervención al igual que los problemas económicos. Estos últimos son otra consecuencia del cuidado debido a la cantidad de recursos materiales y financieros que requiere el cuidar a una persona dependiente; su poca mejoría después de la intervención pudiera estar dado porque es un aspecto muy tangible de la vida del cuidador que resulta más difícil de modificar con intervenciones de tipo psicoeducativas.

Inciden en la problemática económica que el cuidador, tal y como se ve en otro de los resultados del presente estudio, confronta problemas laborales debido a que tiene que ausentarse y dejar de cumplir con estas tareas, llega incluso a abandonar su vínculo laboral o adelanta en muchas ocasiones su jubilación. Esto repercute en la disminución en sus ingresos económicos cuando más lo necesita y por otra parte, limita su vida profesional, interrumpe en ocasiones importantes proyectos en esta esfera y restringe sus relaciones sociales. Ambos aspectos aunque no mejoraron significativamente, hubo un cambio favorable con la intervención, ya que al parecer los cuidadores aprendieron a buscar otras opciones, antes de abandonar el vínculo laboral, no dejándose "aplastar"por la situación.

Se ha enunciado una serie de sentimientos que experimenta el cuidador de la persona que sufre demencia como la ira, la tristeza, la culpa, la vergüenza y el resentimiento entre otros, los cuales influyen negativamente en el cuidado del anciano y pueden dar lugar a alteraciones como la depresión y la ansiedad. Sin embargo existen pocos estudios de intervención donde se enseñe a los cuidadores a 
manejar y controlar adecuadamente estas emociones lo cual pudiera prevenir la aparición de múltiples trastornos, tanto físicos como psicológicos.

En el presente estudio los sentimientos que más manifestaron los cuidadores fueron la angustia, la ira y el miedo lo cual se correspondió con otros estudios realizados. Una investigación encuentra en el $42 \%$ de los cuidadores la aparición de tristeza y deseos de llorar, otro $32 \%$ confiesa tener sentimientos de culpabilidad si no se encontraban al lado del paciente. Las reacciones emocionales adversas publicadas en otro estudio son: ansiedad ( $58 \%$ ), depresión (50\%), miedo (35\%), frustración (32\%), resentimiento ( $29 \%$ ), impaciencia ( $25 \%$ ) y culpabilidad $(10 \%)$, las cuales no se habían diagnosticado ni tratado. Otro estudio revela que el $26,7 \%$ de los cuidadores se sienten solos en el cuidado, el $60 \%$ siente miedo por el futuro, y el $20,7 \%$ tiene depresión. ${ }^{20}$

Con la "Escuela de Cuidadores"se modificó la presencia de los tres sentimientos mencionados. En dicho programa se enseñó al cuidador a manejar la ira como uno de los sentimientos más dañinos para el enfermo, pues da lugar inevitablemente a actitudes de maltrato hacia el anciano y van acompañadas de culpa por lo censurable de dicha conducta hacia alguien "indefenso". Al comprender que su familiar es un enfermo y que toda su conducta se debe a ello, se suprimió en gran medida las ideas de amenaza que dan lugar a la ira, sin embargo los sentimientos de culpa no desaparecieron lo cual pudiera deberse a que este sentimiento está muy vinculado con los lazos afectivos establecidos entre el cuidador y el anciano durante su vida, al igual que el resentimiento que tampoco se modificó después de la intervención.

La angustia y el miedo mejoraron después de la intervención, ya que la información obtenida acerca del manejo adecuado de los síntomas y problemas conductuales de los ancianos, así como el apoyo emocional ofrecido, contribuyeron al mejoramiento del estado de ánimo de dichos cuidadores y les proporcionó más seguridad en el cuidado de su familiar.

Un sentimiento que apenas disminuyó fue la desesperanza, debido posiblemente al conocimiento que adquirieron los cuidadores de la inevitable evolución de la enfermedad y de la ausencia de un tratamiento que detenga el proceso. Es necesario encaminar intervenciones hacia el manejo de este sentimiento tan destructivo para el cuidador, que como es sabido se encuentra en la base de la depresión. Quizás sea conveniente tomar en cuenta lo demostrado en un estudio realizado para evaluar la eficacia de intervenciones psicoeducativas para cuidadores de familiares con demencia, acerca de la importancia de intervenir sobre pensamientos poco adaptativos para un adecuado afrontamiento del cuidado. ${ }^{21}$

De modo general el programa de intervención psicoeducativa no modificó significativamente las afectaciones de índole socioeconómica de los cuidadores, lo que sugiere la incorporación al programa de acciones o estrategias para corregir el afrontamiento ya que las intervenciones realizadas dadas fundamentalmente por información, desarrollo de habilidades y apoyo emocional, no fue suficiente para modificar este tipo de variables. Sin embargo mejoraron en general las afectaciones psicológicas dadas por una reducción de los sentimientos negativos del cuidador.

A partir de los resultados obtenidos se puede decir que todo el proceso de la aplicación del programa fue calificado de satisfactorio, las sesiones se realizaron en su totalidad por los especialistas, con los medios idóneos y la calidad requerida y evaluadas de Muy bien y Bien; se alcanzó buena asistencia y participación de los cuidadores, lo que demostró su elevado grado de satisfacción. Disminuyeron los 
sentimientos negativos de los cuidadores después de la intervención fundamentalmente la ira, la angustia y el miedo. La desesperanza es un sentimiento negativo que se mantuvo después de la intervención debido posiblemente a la pérdida anticipada que experimenta el cuidador. El resentimiento y la culpa son sentimientos que no se modificaron debido posiblemente a que están vinculados a la historia familiar.

Aunque en general disminuyen las afectaciones de tipo socioeconómicas después de la intervención, sobre todo el poco tiempo libre y las dificultades laborales, esta mejoría no es significativa, lo que indica que este tipo de intervención no es suficiente aún para modificar los aspectos más tangibles de la vida del cuidador

Estos resultados demuestran en general que el programa psicoeducativo "Escuela de Cuidadores" es efectivo para modificar la afectación psicológica de los cuidadores, pero no para disminuir las afectaciones de tipo socioeconómicas que produce el cuidado de un anciano con demencia.

\section{Agradecimientos}

la autora agradece la colaboración del equipo de profesionales del Centro I beroamericano para la Tercera Edad integrado por médicos, psicólogos, defectóloga y trabajadoras sociales, que participaron en la aplicación del programa de intervención "Escuela de Cuidadores."

\section{REFERENCI AS BI BLI OGRÁFI CAS}

1. Llibre」J.J. Estrategias de investigación en la enfermedad de Alzheimer. Rev Cubana Med Gen Integr [serie en Internet].2002 [citada Dic 2007]; 18 (4). Disponible en: http://scielo.sld.cu/scielo.php?script=sci_arttext\&pid=S0864$\underline{21252002000400016 \& \operatorname{lng}=\mathrm{es} \& \mathrm{n} r \mathrm{~m}=\mathrm{iso}}$

2. Martínez C, Pérez V, Carballo M, Varona G. Estudio clínico epidemiológico del síndrome demencial. Rev Cubana Med Gen Integr [serie en Internet]. 2005 [citada Dic 2007]; 21(3-4). Disponible en: http: //scielo.sld.cu/scielo.php?script=sci_arttext\&pid=S0864$\underline{21252005000300013 \& \operatorname{lng}=e s \& n r m=i s o}$

3. Wilcock S. The increasing burden of Alzheimer Disease. Alzheimer Dis Associated Disorders. 2003, 17 (supl.3).

4. Stuyck R. La enfermedad de Alzheimer. Impacto sobre cuidadores y sistema sanitario de las personas afectadas. En: Orozco M, Prados N, editores. Temas de Geriatría y Gerontología de I beroamérica. Granada: Imprenta de la Diputación; 2000.p.41-60.

5. Teixidó J, Tartas L, Arias N, Cosculluela A. Cuestionario de sobrecarga de cuidadores de pacientes de diálisis peritoneal. Nefrología. 2006;26(1).

6. Williamson y Shulz. Caring for a familiar member with cancer: past communal behavior and affective reactions. J App Soc Psicol. 1995; 3: 32-9. 
7. Carod FJ, Egido-Navarro JA, González-Gutiérrez JI, Varela de Seijas E. Percepción de la sobrecarga a largo plazo en cuidadores de supervivientes de un ictus. Rev Neurol. 1999; 28(12): 1130-8.

8. Samele C, Manning N. Nivel de carga del cuidador entre los familiares del enfermo mental en el sur de Verona. Eur Psychiatry. 2000; 15: 196- 204.

9. Ginsberg J, Martínez MF, Mendoza A, Pabón J L. Carga subjetiva percibida por el cuidador y su relación con el nivel de deterioro de pacientes con diagnóstico de demencia. Influencia de la edad, estilo de personalidad y tipo de cuidador. Arch Venezolanos Psiquiatría Neurología. 2005; 51(104).

10. Haley WE. Family caregivers of elderly patients with cancer: understanding and minimizing the burden of care. J Support Oncol. 2003; 1(4 Suppl 2):25-9.

11. Asociación de Familiares de Alzheimer (AFA). En casa tenemos un enfermo de Alzheimer. Bilbao: AFA; 1994.

12. Montorio I, Díaz P, Fernández MI. Programas y servicios de apoyo a familiares cuidadores de ancianos dependientes. Rev Esp Geriat. 1995;30(3): 157-68.

13. Sorensen, Pinquart, Duberstein. How effective are interventions with caregivers? An update meta-analysis. The Gerontologist. 2002;42(3):356-72.

14. Proctor A, Martía S, Hewison L. A study of carers' knowledge about dementia, preferred doping style and psychological distress. Interl J Geriatric Psychiatry. 2002; 6: 15-23.

15. Barrera-Ortiz L, Pinto-Afanador N, Sánchez Herrera B. Evaluación de un programa para fortalecer a los cuidadores familiares de enfermos crónicos. Rev Salud Pública. 2006;8(2).

16. Hébert R, Lévesque L, Vézina J, Lavoie JP, Ducharme F, Gendron C, et, al. Efficacy of a psychoeducative group program for caregivers of demented persons living at home. A randomized controlled trial. Centre on Aging, Sherbrooke Geraitric University Institute Québec. Canadá: Centre; 1999.

17. Espín AM, Seco T. Metodología de intervención educativo-terapéutica en cuidadores de ancianos con demencia de Alzheimer. Rev Arg Geriatría. 1998; 18:2753.

18. Perlado F. Apoyo a los cuidadores de enfermos de Alzheimer. Rev Gerontol. 1995; 10: 7-53.

19. Roig MV, Abengózar MC, Serra E. La sobrecarga en los cuidadores principales de enfermos de Alzheimer. Anales Psicología. 1998; 14(2):215-27.

20. Anderson CS, Linto J, Stewart Wynne EG. A population-based assessment of the impact and burden of caregiving for long-term stroke survivors. Stroke. 1995; 26: 843-9.

21. Losada A, Izal M, Montorio I, Márquez M, Pérez G. Eficacia diferencial de dos intervenciones psicoeducativas para familiares con demencia. Rev Neurol. 2004; 38(8): 701. 
Recibido: 29 de noviembre de 2007.

Aprobado: 2 de junio de 2008.

Ana Margarita Espín Andrade. Escuela Latinoamericana de Medicina (ELAM). La Habana, Cuba.

E-mail: amespin@infomed.sld.cu 